separately associated with higher BMI z-score (3 years: 0.11, 95\% CI 0.03 to 0.19 ; 5 years: $0.13,0.05$ to 0.20 ; 11 years: $0.16,0.05$ to 0.27 ) and the presence of overweight at 11 years. Current informal care had the strongest association, however, informal child care at 5 years also made a contribution. There was no evidence of differences by sex or SEP.

Conclusions In a developed non-western setting, informal child care was associated with childhood obesity. Modifiable attributes of informal child care warrant investigation for obesity prevention.

\section{P1-219 MUSLIMS, HINDUS AND SIKHS ACCESS TO NHS SERVICES IN SCOTLAND}

doi:10.1136/jech.2011.142976e.12

'J G Love, ${ }^{* 1,2} \mathrm{~K}$ Levecque, ${ }^{1,2} \mathrm{R}$ Van Rossen, ${ }^{1,3} \mathrm{E}$ Ronda. ${ }^{1}$ Robert Gordon University, Aberdeen, UK; ${ }^{2}$ Ghent University, Ghent, Belgium; ${ }^{3}$ Alicante University, Alicante, Spain

Introduction Scotland is a multi-cultural society with $2 \%$ of the population made up of people from a minority ethnic background, more than $70 \%$ of whom are Asian and from a Pakistani, Indian, Bangladeshi or other South Asian background. Such a mix of people from minority ethnic backgrounds gives rise to a multitude of different needs and expectations that require to be incorporated within health service planning and delivery.

Objectives Muslims generally experience some of the poorest health in the UK. The present study (2008-2011), funded by the Scottish Health Council, sought to examine, through comparative analysis with Hindus (and a small number of Sikhs), the extent to which NHS services engaged with Muslims.

Methods The research, based on a mixed methods approach combined a survey $(n=111)$ and focus group discussions with Muslims, Hindus and Sikhs living in three of Scotland's major cities: Aberdeen, Dundee and Glasgow.

Results The study found heightened levels of poor general health coupled with high levels of satisfaction with NHS services among study participants. Making greater use of GP services and similar use of hospital out-patient services, individual factors (eg, knowledge, experience) were positively implicated in service use while organisational factors (eg, waiting lists) inhibit such use. Although most participants did not perceive there to be a problem of religious or ethnic discrimination in the NHS, a fifth disagreed, with Muslims more likely to do so.

Conclusion Improvements are needed to ensure fair and adequate access to healthcare is provided to minority ethnic groups in Scotland.

\section{P1-220 HOW DO IN-HOSPITAL AND 30-DAY POST DISCHARGE HOSPITAL MORTALITY MEASURES COMPARE? AN AUSTRALIAN PERSPECTIVE}

doi:10.1136/jech.2011.142976e.13

${ }^{1} \mathrm{~S}$ Lujic, ${ }^{* 1,2} \mathrm{~L}$ Jorm, ${ }^{1} \mathrm{D}$ Randall. ${ }^{1}$ University of Western Sydney, Sydney, New South Wales, Australia; ${ }^{2}$ Sax Institute, Sydney, New South Wales, Australia

Introduction The hospital standardised mortality ratio (HSMR) has been used internationally to help support improvements in hospital quality of care. Most commonly, HSMRs are computed using only in-hospital deaths, influenced by length of stay. Following a major review, the National Health Service in England recommended that HSMR calculations include both in- and 30-day post-discharge mortality. Our study investigated the relationship between these two measures.

Methods Retrospective analysis of probabilistically linked mortality and hospital data for patients admitted to 162 hospitals in New
South Wales, Australia between July 2000 and June 2008. HSMRs for in-hospital and 30-day post discharge (30-day HSMR) were calculated by dividing an observed number of deaths by the logisticregression derived expected number and compared using correlation coefficients and outlier status.

Results There were 270456 hospital admissions ending in death either in-hospital (147926; 55\%) or up to 30 days post discharge (122 530; 45\%). HSMRs ranged from 12.5 to 251.7 for in-hospital HSMR and 14.3 to 195.4 for 30-day HSMR. Correlation between the two measures was high (Spearman $\rho=0.868, p<0.001$ ) but there was moderate agreement on outlier status $(\kappa=0.58)$. Five hospitals swapped status between significantly higher/lower HSMR of 100.

Conclusions In-hospital and 30-day HSMRs were similar across hospitals, even though there were differences in agreement on outlier status and whether HSMRs 'signalled' as significantly high or low. 30-day HSMRs are more difficult to calculate, and may present few advantages over conventional HSMRs where the aim is to initiate more detailed investigation of possible hospital performance issues.

\section{P1-221 EPIDEMIOLOGY OF DEPRESSION IN OLD AGE: RESULTS OF THE LEIPZIG LONGITUDINAL STUDY OF THE AGED (LEILA 75+)}

doi:10.1136/jech.2011.142976e.14

${ }^{1} \mathrm{M}$ Luppa, ${ }^{*} \mathrm{C}$ Sikorski, ${ }^{1,2} \mathrm{~T}$ Luck, ${ }^{3} \mathrm{~S}$ Weyerer, ${ }^{4} \mathrm{~A}$ Villringer, ${ }^{5}$ Hans-Helmut König ${ }^{6} S$ G Riedel-Heller. ${ }^{1}$ Institute of Social Medicine, Occupational Health and Public Health, Public Health Research Unit, University of Leipzig, Leipzig, Germany; ${ }^{2}$ LIFELeipzig Research Center for Civilisation Diseases, University of Leipzig, Leipzig, Germany; ${ }^{3}$ Central Institute of Mental Health, Mannheim, Germany; ${ }^{4}$ Max Planck Institute for Human Cognitive and Brain Sciences and Day Clinic of Cognitive Neurology, University of Leipzig, Leipzig, Germany; ${ }^{5}$ Department of Medical Sociology and Health Economics, University Medical Center Hamburg-Eppendorf, Hamburg, Germany; ${ }^{6}$ Institute of Social Medicine, Occupational Health and Public Health, Leipzig, Germany

Background Depression is one of the most common mental disorders in old age. In order to assess future needs of the healthcare system for prevention and treatment, information on epidemiology of depression among the highest age groups is required. However, most previous studies just focused on prevalence and incidence rates of late life depression across the entire old age.

Methods For a population-based sample of 1265 elderly individuals aged 75 years and older, prevalence and incidence rates as well as risk factors of depression were determined. Individuals were requested every 1.5 years over six waves. Depression was assessed dimensionally by the CES-D (Center of Epidemiologic Studies Depression Scale) and categorically by the SCID (Structured Clinical Interview for DSM-IV). The prevalence rates were $1.0 \%$ for Major Depression, 2.5\% for Minor Depression according to DSM-IV and $38.2 \%$ for depressive symptoms according to CES-D. The rates increased for Minor and depressive symptoms with rising age. Risk factors were divorced or widowed marital status, low educational level, poor self-rated health status, stressful life events, and poor social network. The incidence rates were 6.9 per 1000 person-years (py) for Major Depression, 16.6 per 1000 py for Minor Depression and 33.9 per 1000 py for depressive symptoms.

Discussion Since depressive symptoms are common in oldest age and associated with broad categories of risk factors, latest-life depression represents an important public health issue. Employment of comprehensive geriatric assessment to ascertain depressive symptoms and its concomitants could help to improve treatment success. 